\title{
Seminoma in the Testis Presenting as Hemospermia
}

\author{
Sami Beji ${ }^{a} \quad$ Martin Hoejgaard $^{b} \quad$ Peter Lyngdorf $^{a}$ \\ ${ }^{a}$ Department of Urology, Naestved Hospital, Naestved, and \\ ${ }^{\mathrm{b}}$ Department of Urology, Herlev Hospital, Herlev, Denmark
}

\section{Key Words}

Seminoma $\cdot$ Hemospermia $\cdot$ Testis

\begin{abstract}
Hemospermia is often considered idiopathic. We report a case of a patient who presented with hemospermia. Scrotal examination and ultrasonography found a testis tumor. This case underscores the importance of scrotal examination and eventually ultrasound in patients presenting with hemospermia.
\end{abstract}

\section{Introduction}

Hemospermia is defined as the macroscopic presence of blood in semen and is often considered idiopathic. The number of cases where hemospermia is considered idiopathic is decreasing due to new diagnostic methods.

\section{Case Report}

A 49-year-old man presented to his general practitioner with a history of 4 episodes of monosymptomatic hemospermia in a period of 2 months. The patient did not notice any tumor or pain in the testes. On clinical examination, the general practitioner found a palpable tumor at the upper pole of the right testis. Ultrasonography showed a hypoechoic area on the upper pole of the testis (fig. 1). Abdominal and chest CT showed no metastases and the patient underwent orchiectomy. Microscopy showed a 9-mm pT2 seminoma limited to the testis with vascular invasion but no invasion of the tunica albuginea, epididymis or funiculus. Tumor cells stained positive for PLAP, D240 and CD117, and stained negative for HCG, AFP and CD30.

Written informed consent was obtained from the patient for publication of this case report and accompanying images. A copy of the written consent is available for review by the Editor-in-Chief of this journal. 


\section{Discussion}

Current recommendations state that most patients presenting with hemospermia can be treated with minimal investigation and simple reassurance [1]. A prospective study concluded that an investigation following the discovery of hemospermia should include a focused history, clinical examination including a digital rectal examination, serum PSA, urine cultures and screening for sexually transmitted diseases [2]. Ultrasonography of the scrotum in that study was not performed as an investigational method for hemospermia. Previously, Kumar et al. [3] suggested an algorithm for hemospermia investigation that did not include scrotal ultrasound for patients below 40 years of age with recurrent hemospermia. An algorithm for hemospermia by the American Academy of Family Physicians recommends scrotal examination for patients below 40 years of age presenting with hemospermia to rule out infection and testicular cancer [4]. The first case of testicular cancer presenting with hemospermia was reported in 2001 [5], and 2 other cases were reported in 2002 [6]. In all 3 cases, scrotal examination and ultrasonography were performed.

Our patient had no history of prostatic disease, infections or systemic diseases which could explain the hemospermia, making the seminoma the most likely cause of the bleeding.

Testicular cancer is most common in young men and can, in many cases, be diagnosed by scrotal examination alone. As the procedure is noninvasive and therapeutic options exist for the above-mentioned causes, we recommend scrotal examination in patients presenting with hemospermia to exclude testicular tumors or infections of the scrotal contents. We further recommend ultrasound investigation of the scrotal contents if testicular tumors are found on clinical examination, as recommended in the current guidelines [7].

\section{Conclusion}

Hemospermia is often considered to be a symptom of little significance, and its etiology is poorly understood. This case of monosymptomatic hemospermia in a man with seminoma underscores the importance of scrotal examination and eventually ultrasonography in such patients. The discovery of seminoma in this case may only be incidental, but scrotal examination is easy, noninvasive and should be performed in all patients presenting with hemospermia.

\section{Disclosure Statement}

The authors declare that they have no competing interests. 


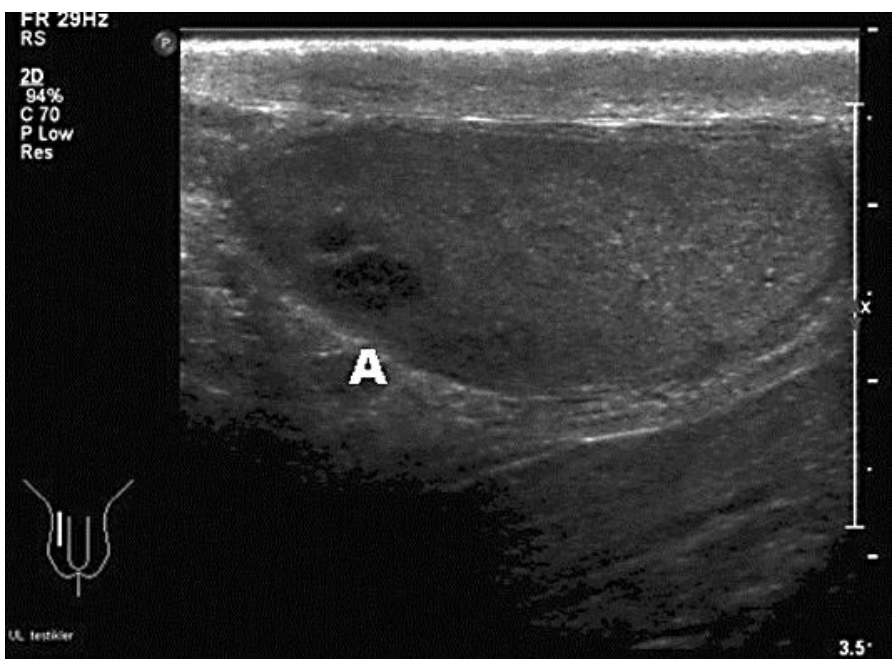

Fig. 1. Scrotal ultrasound image of the right testis in the current case. The hypoechoic area above the 'A' represents the seminoma.

\section{References}

1 Ahmad I, Krishna NS: Hemospermia. J Urol 2007; 177:1613-1618.

-2 Kumar AA, Zachariah KK, Dorkin TJ: Is there any value investigating persistent haematospermia? Results of a 12-year prospective study. Br J Med Surg Urol 2011;4:202-206.

-3 Kumar P, Kapoor S, Nargund V: Haematospermia - a systematic review. Ann R Coll Surg Engl 2006;88:339-342.

4 Stefanovic KB, Gregg PC, Soung M: Evaluation and treatment of hematospermia. Am Fam Physician 2009;80:1421-1427.

-5 Maheshkumar P, Otite U, Gordon S, Berney DM, Nargund VH: Testicular tumor presenting as hematospermia. J Urol 2001;165:188.

6 Vilandt J, Sonksen J, Mikines K, Torp-Pedersen S, Colstrup H: Seminoma in the testes associated with haemospermia. BJU Int 2002;89:633.

-7 Albers P, Albrecht W, Algaba F, Bokemeyer C, Cohn-Cedermark G, Fizazi K, et al: EAU guidelines on testicular cancer: 2011 update. Eur Urol 2011;60:304-319. 